\title{
Immunotherapy Targeting Pathological Tau Prevents Cognitive Decline in a New Tangle Mouse Model
}

\author{
Allal Boutajangout, ${ }^{1,2}$ David Quartermain, ${ }^{1,3}$ and Einar M. Sigurdsson ${ }^{1,2}$ \\ Departments of ${ }^{P}$ Physiology and Neuroscience, ${ }^{2}$ Psychiatry, and ${ }^{3}$ Neurology, New York University School of Medicine, New York, New York 10016
}

Harnessing the immune system to clear protein aggregates is emerging as a promising approach to treat various neurodegenerative diseases. In Alzheimer's disease (AD), several clinical trials are ongoing using active and passive immunotherapy targeting the amyloid- $\beta$ $(\mathrm{A} \beta)$ peptide. Limited emphasis has been put into clearing tau/tangle pathology, another major hallmark of the disease. Recent findings from the first $\mathrm{A} \beta$ vaccination trial suggest that this approach has limited effect on tau pathology and that $\mathrm{A} \beta$ plaque clearance may not halt or slow the progression of dementia in individuals with mild-to-moderate AD. To assess within a reasonable timeframe whether targeting tau pathology with immunotherapy could prevent cognitive decline, we developed a new model with accelerated tangle development. It was generated by crossing available strains that express all six human tau isoforms and the M146L presenilin mutation. Here, we show that this unique approach completely prevents severe cognitive impairment in three different tests. This remarkable effect correlated well with extensive clearance of abnormal tau within the brain. Overall, our findings indicate that immunotherapy targeting pathological tau is very feasible for tauopathies, and should be assessed in clinical trials in the near future.

\section{Introduction}

Immunotherapies targeting the amyloid- $\beta(\mathrm{A} \beta)$ peptide in Alzheimer's disease $(\mathrm{AD})$ are currently in several clinical trials, with a few having advanced into phase III based on some promising findings (Kerchner and Boxer, 2010). In the AN-1792 trial, A $\beta$ plaque clearance had limited effect on tau pathology (Nicoll et al., 2003; Ferrer et al., 2004; Masliah et al., 2005a; Holmes et al., 2008; Boche et al., 2010a,b; Serrano-Pozo et al., 2010), which emphasizes the need for therapy that specifically targets this other major hallmark of the disease. Furthermore, recent findings from this trial indicate that plaque clearance did not appear to halt or slow the progression of dementia once it was well underway, suggesting that alternative targets are needed at this stage of the disease (Holmes et al., 2008). Targeting A $\beta$ and tau simultaneously should also improve therapeutic efficacy because these pathologies are likely synergistic (Sigurdsson, 2009). Recent reports that extracellular tau is important for the anatomical spread of tau pathology strengthen as well the feasibility of effectively reducing these lesions (Clavaguera et al., 2009; Frost et al., 2009).

A key feature of any promising experimental treatment for $\mathrm{AD}$ is to prevent or attenuate cognitive decline. This issue has been difficult to assess in available tangle mouse models, either because of their tangle-related motor impairments or late onset of tau pathology. Therefore, we developed a tangle model for cognitive

Received Aug. 19, 2010; revised 0ct. 1, 2010; accepted 0ct. 6, 2010.

This work was supported by National Institutes of Health Grants AG032611 and AG020197, the Alzheimer's Association, and the Irma T. Hirschl Charitable Trust. New York University patent pending on tau immunotherapy. We thank Oligomerix Inc. for providing purified recombinant tau protein.

Correspondence should be addressed to Dr. Einar M. Sigurdsson, Associate Professor of Physiology and Neuroscience, and Psychiatry, NYU School of Medicine, Medical Science Building, Room MSB459, 550 First Avenue, New York, NY 10016. E-mail: einar.sigurdsson@nyumc.org.

DOI:10.1523/JNEUROSCI.4363-10.2010

Copyright $\odot 2010$ the authors $\quad 0270-6474 / 10 / 3016559-08 \$ 15.00 / 0$ testing by crossing htau mice (Andorfer et al., 2003) with a model carrying the human presenilin 1 (PS1) M146L mutation (Duff et al., 1996). The htau mice express unmutated human tau without mouse tau, and the new model was maintained on a mouse tau knock-out background. These mice have an earlier onset, at or before 2 months of age, and more rapid progression of tau pathology than the htau mice, while the distribution is similar with extensive involvement of hippocampal and cortical regions. These features render this model ideally suited for efficient screening of tau-targeting therapy.

Here, we report that tau immunotherapy prevents cognitive decline in several tests in the htau/PS1 model that was associated with reduction in pathological tau within the brain.

\section{Materials and Methods}

Peptides and recombinant tau protein

Tau peptides were synthesized and purified at the Keck facility (Yale University) as described previously (Sigurdsson et al., 2001). Highly purified full-length human Tau441 (2N/4R) was generously provided by Oligomerix.

\section{Mice}

The htau model (Jackson Laboratories, stock \#004808) (Andorfer et al., 2005) was crossed with a model that expresses the PS1 M146L human mutation (Duff et al., 1996). The htau mice express unmutated human tau protein on a null mouse tau background and develop tau pathology and tangles with age. The new htau X PS1 model on a mouse tau knockout background (mtau KO), referred to as htau/PS1, has an earlier onset and more aggressive progression of tau pathology than the htau model. The mice (3-4 months of age) received $100 \mu \mathrm{g}$ of Tau379-408[P-Ser $\left.{ }_{396,404}\right]$ intraperitoneally in $100 \mu$ l of alum adjuvant (Adju-Phos, Brenntag Biosector) with the first three injections every 2 weeks. Subsequent administration was at monthly intervals. The peptide was mixed with the adjuvant overnight at $4^{\circ} \mathrm{C}$ to allow it to adsorb onto the aluminum phosphate adjuvant. The control groups received adjuvant alone. Those were (1) htau/PS1, (2) htau/PS1/mtau, and (3) htau mice. As described above, 
the htau/PS1 and htau mice are on an mtau KO background. At 7-8 months, the mice went through extensive behavioral testing and were subsequently killed for tissue analyses at 8-9 months of age. All the mouse lines were on the same genetic background as they were littermates from the same colony. According to Jackson Laboratories, the htau mice that we received had been maintained on a BL6 $\times 129$ S4 $\times$ SW $\times$ DBA2/J background. The PS1 Tg mice were originally SW $\times$ B6D2F1 and then bred for several years on a BL6/SJL background. We decided to add the htau/PS1/mtau and htau groups as additional controls because our preliminary analysis indicated that these models had less pathology than the htau/PS1 model.

\section{Antibody response}

The antibody response was determined by 1:200 dilution of plasma using an ELISA assay as we have described previously (Sigurdsson et al., 2001, 2004), in which the immunogen, its unphosphorylated sequence, or the full-length recombinant tau peptide were coated onto microtiter wells (Immulon 2HB, Thermo Electron). The signal was detected by a goat anti-mouse IgG or IgM linked to a horseradish peroxidase (Pierce), and tetramethyl benzidine (Pierce) was the substrate. The mice were bled prior to immunization (T0), 1 week after the third (T1) and fourth (T2) injection, and at the end of the study (Tf).

\section{Histology}

Following behavioral testing, mice were deeply anesthetized with ketamine/xylazine ( $250 \mathrm{mg} / 50 \mathrm{mg}$ per kg body weight, i.p.). The brain was subsequently removed without perfusion and processed as previously described (Sigurdsson et al., 1996). The left hemisphere was snap frozen and stored at $-80^{\circ} \mathrm{C}$ until processed for Western blots. Coronal brain sections $(40 \mu \mathrm{m})$ of the right hemisphere were saved for histological staining with the following: (1) mouse monoclonal tau antibodies that stain pathological tau PHF1 [1:1000, recognizes phosphorylated serines 396 and 404 of tau protein (Otvos et al., 1994), and shows only minimal axonal staining in normal human brain, generously provided by Peter Davies (Albert Einstein College of Medicine, New York, NY)] and AT8 [1:500, recognizes tau phosphorylated at serine 202 and adjacent sites (Goedert et al., 1993), Pierce]; (2) rabbit polyclonal antibody against glial fibrillary acidic protein (GFAP 1:500, Dako) in astrocytes; and (3) tomato lectin $(10 \mu \mathrm{g} / \mathrm{ml}$, Vector Laboratories) to detect microglia. The sectioned series were placed in ethylene glycol cryoprotectant and stored at $-20^{\circ} \mathrm{C}$ until used for immunohistochemistry.

Staining was performed as previously described (Sigurdsson et al., 1996, 2001; Asuni et al., 2006), using mouse-on-mouse immunodetection kit (Vector Laboratories) for the monoclonals.

To verify that reduced tau immunoreactivity in immunized mice was not caused by epitope masking, unmasking procedure was performed as described previously (Asuni et al., 2007).

\section{Image analysis}

Tau pathology in brain sections was quantified blindly with the Bioquant system as described previously (Asuni et al., 2007). Every 10th section, randomly chosen, was stained with each antibody. The measurement was the percentage of area in the measurement field $(200 \times)$ of the pyriform cortex that was occupied by reaction product. This brain region was chosen as it contained prominent tau pathology. Five sections were analyzed per animal.

\section{Rating of microgliosis and astrogliosis}

The assessment of the tomato lectin (microglia)-stained sections was based on a semiquantitative analysis of the extent of microgliosis throughout the brain ( 0 , predominantly resting microglia; $1+$, a few ramified and/or phagocytic microglia; $2+$, moderate number of ramified/phagocytic microglia; $3+$, numerous ramified/phagocytic microglia). The rating of the GFAP sections was based on the complexity of astrocytic branching throughout the brain $(1+$, resting astrocytes, few processes; $2+$, reactive astrocytes, moderate branching; $3+$, reactive astrocytes, extensive branching).

\section{Western blotting}

Brain tissue was homogenized in a buffer containing $0.1 \mathrm{~mm} 2-(\mathrm{N}-$ morpholino) ethanesulfonic acid, $0.5 \mathrm{~mm} \mathrm{MgSO}_{4}, 1 \mathrm{~mm}$ EGTA, $2 \mathrm{~mm}$ dithiothreitol, $\mathrm{pH}$ 6.8, $0.75 \mathrm{~mm} \mathrm{NaCl}, 2 \mathrm{~mm}$ phenylmethyl sulfonyl fluoride, Complete mini protease inhibitor mixture $(1$ tablet in $10 \mathrm{ml}$ of water; Roche), and phosphatase inhibitors ( $20 \mathrm{~mm} \mathrm{NaF}$ and $0.5 \mathrm{~mm}$ sodium orthovanadate). The homogenate was then centrifuged $(20,000 \times$ g) for $30 \mathrm{~min}$ at $4^{\circ} \mathrm{C}$ to separate a soluble cytosolic fraction (supernatant 1 ) and insoluble fraction (pellet 1). The pellet was resuspended in the same volume of buffer without protease and phosphatase inhibitors, but that contained $1 \%(\mathrm{v} / \mathrm{v})$ Triton X-100 and $0.25 \%(\mathrm{w} / \mathrm{v})$ deoxycholate sodium and ultracentrifuged at 50,000 for $30 \mathrm{~min}$ to obtain a detergentextracted supernatant 2 that was analyzed as insoluble fraction. Supernatant 1 and 2 were heated at $100^{\circ} \mathrm{C}$ for $5 \mathrm{~min}$, and the same amount of protein was electrophoresed on $12 \%(\mathrm{w} / \mathrm{v})$ polyacrylamide gel. The blots were blocked in $5 \%$ nonfat milk with $0.1 \%$ Tween-20 in TBS, and incubated with different antibodies overnight, and then washed and incubated at room temperature for $1 \mathrm{~h}$ with peroxidase-conjugated, anti-mouse, or anti-rabbit IgG. Subsequently, the bound antibodies [PHF1, CP13 (P-Ser202, generously provided by Peter Davies, B19 (total tau, generously provided by JeanPierre Brion, Free University of Brussels, Brussels, Belgium), anti-actin (Sigma)] were detected by ECL (Pierce). Densitometric analysis of immunoblots was performed by National Institutes of Health ImageJ program, and the levels of pathological tau were normalized relative to actin and total tau protein.

\section{Behavioral studies}

The objective was to evaluate the effects of the vaccination on selected sensorimotor and cognitive behaviors. The animals were tested in the month before and at tissue harvesting at the end of the study.

The htau model was recently reported to develop cognitive deficits with age (Polydoro et al., 2009), but the M146L PS1 model does not appear to develop memory impairments (Sadowski et al., 2004). Our main focus was on cognitive testing in the htau/PS1 model to determine if (1) it had impairments in learning and memory, and (2) if the immunotherapy targeting pathological tau would prevent or attenuate possible cognitive deficits. Sensorimotor tests were used to verify that any measured differences in cognition could not be explained by poor motor performance and hence limited maze navigation. The tests were as follows: (1) locomotor activity; (2) motor and reflex behaviors, including traverse beam test and accelerating rotarod; and (3) learning and memory tests, including radial maze learning and retention, closed-field symmetrical maze, and object recognition. We routinely use all these tests in our mouse immunotherapy studies, using procedures previously described (Sigurdsson et al., 2004; Asuni et al., 2006, 2007).

\section{Sensorimotor tests}

Before testing, the mice were adapted to the room with lights on for $15 \mathrm{~min}$.

\section{Locomotor activity}

Exploratory locomotor activity was recorded in a circular open field activity chamber $(70 \mathrm{~cm}$ in diameter). A video camera mounted above the chamber automatically recorded horizontal movements in the open field in each dimension (i.e., $x$-, $y$-, and two $z$-planes; San Diego Instruments). Total distance was measured in centimeters traveled and is defined as sequential movement interruptions of the animal (white) measured relative to the background (black). The duration of the behavior was timed for $15 \mathrm{~min}$. Results are reported based on distance traveled (in centimeters), mean resting time, and velocity [mean $\left(V_{\text {mean }}\right)$ and $\left.\operatorname{maximum}\left(V_{\max }\right)\right]$ of the animal.

\section{Rotarod}

The animals were placed onto the rod (diameter $3.6 \mathrm{~cm}$ ) apparatus to assess differences in motor coordination and balance by measuring forelimb and hindlimb motor coordination and balance (Rotarod $7650 \mathrm{ac}-$ celerating model; Ugo Basile). This procedure was designed to assess motor behavior without a practice confound. The animals were habituated to the apparatus by receiving training sessions in two trials, sufficient to reach a baseline level of performance. Then the mice were tested a further three times, with increasing speed. During habituation, the rotarod was set at $1.0 \mathrm{rpm}$, which was gradually raised every $30 \mathrm{~s}$, and was also wiped clean with $30 \%$ ethanol solution after each session. A soft foam cushion was placed beneath the apparatus to prevent potential injury from falling. Each animal was tested for three sessions, with each 
session separated by $15 \mathrm{~min}$, and measures were taken for rotarod speed when the animals fell or inverted (by clinging) from the top of the rotating barrel.

\section{Traverse beam}

This task tests balance and general motor coordination and function integration. Mice were assessed by measuring their ability to traverse a narrow wooden beam to reach a goal box. The mice were placed on a $1.1-\mathrm{cm}$-wide beam that is $50.8 \mathrm{~cm}$ long and suspended $30 \mathrm{~cm}$ above a padded surface by two identical columns. Attached at each end of the beam is a shaded goal box. Mice were placed on the beam in a perpendicular orientation to habituate and were then monitored for a maximum of $60 \mathrm{~s}$. The number of foot slips each mouse had before falling or reaching the goal box was recorded for each of four successive trials. Errors were defined as footslips and were recorded numerically. To prevent injury from falling, a soft foam cushion was always kept underneath the beam. Animals that fell off were placed back in their position before the fall.

\section{Learning and memory tests}

Radial arm maze. The apparatus is an eight arm elevated radial maze constructed from Plexiglas. Each arm is $35 \mathrm{~cm}$ long and $7 \mathrm{~cm}$ wide with a water cup $1 \mathrm{~cm}$ in diameter positioned at the end of each arm. Sidewalls $15 \mathrm{~cm}$ high extend $12 \mathrm{~cm}$ into each arm to prevent animals from crossing between arms. The central area is an octagonal-shaped hub $14 \mathrm{~cm}$ in diameter. Clear Plexiglas guillotine doors, operated remotely by a pulley system, control access to the arms. The maze is elevated $75 \mathrm{~cm}$ above floor level and situated in a room in which several distinctive objects of a constant location serve as extra maze cues. Before testing, mice were adapted for $5 \mathrm{~d}$. During this period, the mice received $0.1 \%$ saccharine in water for $1 \mathrm{~h} / \mathrm{d}$ and were then adapted $16 \mathrm{~h}$ later to access the sugar solution from a cup placed at the end of each arm. The first $2 \mathrm{~d}$ of adaptation were performed in a Y-maze, which the mice were allowed to explore freely. The subsequent $3 \mathrm{~d}$ of adaptation were performed in the radial arm maze (RAM), in which the doors were raised and lowered periodically to accustom the animals to the sound associated with their operation. The same water-deprivation schedule was maintained during the $9 \mathrm{~d}$ testing period. The mice maintain good health on this schedule. Each testing trial was begun by placing the mouse in the central area and raising all doors. When an arm was entered, all doors were lowered. After the mouse consumed the saccharine water, the door to that arm was raised, allowing the mouse to return to the central arena. After a $5 \mathrm{~s}$ interval, the next trial was initiated by again raising all of the doors simultaneously. This procedure was continued until the animal had entered all eight arms or until $10 \mathrm{~min}$ had elapsed. Daily acquisition sessions were continued for $9 \mathrm{~d}$. The number of errors (entries to previously visited arms) and the time to complete each session were recorded.

Closed field symmetrical maze. This apparatus is a rectangular field 30 $\mathrm{cm}$ square with $9 \mathrm{~cm}$ high walls divided into $369.5 \mathrm{~cm}$ squares and covered by a clear Plexiglas top. End boxes, each $11 \times 16 \times 9 \mathrm{~cm}$, are situated at diagonal corners of the field. The symmetrical maze is a modification of the Hebb-Williams and Rabinovitch-Rosvold types of tests, as we have discussed previously (Asuni et al., 2006). Briefly, the main difference is that each end compartment functions as both a start box and a goal box, and the mice run in opposite directions on alternate trials, thereby eliminating intertrial handling. The barriers are placed in the field in symmetrical patterns, so that mice face the same turns going in either direction within a given problem. Before testing, the mice were adapted to a water-restriction schedule ( $2 \mathrm{~h}$ daily access to water). The mice were given two adaptation sessions before the beginning of testing. In the first session, all animals were given saccharine-flavored water in the goal box for $10 \mathrm{~min}$. In session 2, they were placed in the start chamber and permitted to explore the field and enter the goal box where water reward $(0.05 \mathrm{ml})$ was available. When the mice were running reliably from the start chamber to the goal box, they were given three practice sessions on simple problems where one or two barriers were placed in different positions in the field so as to obstruct direct access to the goal box. Formal testing consisted of the presentation of three problems graded for difficulty, based on our data (Asuni et al., 2006) and published norms for mice. One problem was presented per day, and the mice were given five trials on each problem with an intertrial interval of 2 min. Performance was scored manually by the same observer in terms of errors (i.e., entries and reentries into designated error zones) and time to complete each trial.

Object recognition. The spontaneous object recognition test (ORT) that was used measures deficits in short term memory, and was conducted in a square-shaped open field box ( $48 \mathrm{~cm}$ square, with $18 \mathrm{~cm}$ high walls constructed from black Plexiglas), raised $50 \mathrm{~cm}$ from the floor. The light intensity was set to 30 lux. On the day before the tests, mice were individually habituated in a session in which they were allowed to explore the empty box for $15 \mathrm{~min}$. During training sessions, two novel objects were placed at diagonal corners in the open field and the animal was allowed to explore for $15 \mathrm{~min}$. For any given trial, the objects in a pair were $10 \mathrm{~cm}$ high and composed of the same material so that they could not readily be distinguished by olfactory cues. The time spent exploring each object was recorded by a tracking system (San Diego Instruments), and at the end of the training phase the mouse was removed from the box for the duration of the retention delay $(3 \mathrm{~h})$. Normal mice remember a specific object after a delay of $3 \mathrm{~h}$ and spend the majority of their time investigating the novel object during the retention trial. During retention tests, the animals were placed back into the same box, in which one of the previous familiar objects used during training was replaced by a novel object, and allowed to explore freely for $6 \mathrm{~min}$. A different object pair was used for each trial for a given animal, and the order of exposure to object pairs as well as the designated sample and novel objects for each pair were counterbalanced within and across groups. The time spent exploring the novel and familiar objects was recorded for the $6 \mathrm{~min}$. The percentage short-term memory score is the time spent exploring any one of the two objects (training session) compared with the novel one (retention session).

\section{Data analysis}

All the data were analyzed with GraphPad Prism 5.01, except for the radial arm maze data, which were analyzed with Statistica 6 . The amount of tau aggregates on Western blots, the immunoreactivity on brain sections within the pyriform cortex, the locomotor activity measurements (distance, $V_{\max }, V_{\text {mean }}$, rest time), and the object recognition test were analyzed by one-way ANOVA test and Newman-Keuls post hoc test. When the data failed at least two of three normality tests (KolmogorovSmirnov, D'Agostino and Pearson omnibus, and Shapiro-Wilk normality tests), a nonparametric Kruskal-Wallis test was used, followed by Dunn's post hoc analysis. The data from the radial arm maze, traverse beam, and rotarod were analyzed by two-way ANOVA repeated measures and a Newman-Keuls (Statistica) or a Bonferroni (GraphPad) post hoc test. Correlation between behavioral outcomes and tau pathology, as assessed by immunohistochemistry or Western blotting, was analyzed by Pearson $r$ correlation or Spearman rank correlation if the data failed at least two of three normality tests.

Besides significant cognitive differences between treated and control htau/PS1 mice in the closed field symmetrical maze (CFSM), post hoc analysis also revealed significant differences between some of the other groups, but those differences are not relevant to the tau immunotherapy and are therefore not detailed.

\section{Results}

Mice immunized with the Tau379-408[P-Ser $\left.{ }_{396,404}\right]$ immunogen in alum adjuvant developed good IgG antibody response against the immunogen (Fig. $1 A$ ). Those antibodies recognized its unphosphorylated version as well (Fig. $1 A$ ). The immunogen was selected based on its overall immunogenicity and its $\mathrm{AD}$ phospho-epitope. It was therefore expected that the polyclonal response would include antibodies against regions outside the phospho-epitope. The following three nonimmunized control groups were included that received adjuvant alone: (1) identical htau/PS1 mice on a mouse tau knock-out background; (2) htau/ PS1 mice that expressed mouse tau (htau/PS1/mtau); and (3) htau littermates without mouse tau. Low binding to recombinant tau was observed in both controls and immunized mice, compa- 
rable to control levels against the immunogenic epitope. We have previously detected such autoantibodies in the JNPL3 P310L model (Asuni et al., 2007). IgM response was less pronounced, as expected, but was of a similar pattern as the IgG response. Plasma from the immunized mice recognized tau pathology in $\mathrm{AD}$ and mouse tissue (data not shown), as we observed previously with this immunogen in a different mouse model (Asuni et al., 2007).

The htau/PS1 mice were clearly cognitively impaired based on our historical norms for wild-type mice in these tests. The other transgenic control groups showed equal or lesser deficits, with the htau/PS1/mtau group showing less overall decline than the htau group. The tau immunotherapy prevented cognitive decline in all three tests that were employed: (1) the RAM (two-way ANOVA repeated measures, $p<0.0001$ ) (Fig. $1 B$ ); (2) the ORT (one-way ANOVA, $p=0.005$ ) (Fig. $1 C)$; and (3) the CFSM (one-way ANOVA: maze I, $p<0.001$; maze II, $p<$ 0.0001; maze III, $p<0.01$ ) (Fig. $1 D$ ), all of which we have used extensively in other models (Sigurdsson et al., 2004; Asuni et al., 2006, 2007). In the RAM and the CFSM, the immunized htau/PS1 mice performed better than the control htau/ PS1 mice on all the days (RAM; $p<0.01-$ 0.001 ) and in all the mazes that were of increasing complexity, as indicated by the number of errors (note that the $y$-axis scale differs; CFSM: maze I, $p<0.01$; mazes II and III, $p<0.001)$. In the ORT, post hoc analysis revealed that the immunized htau/PS1 mice had better shortterm memory than identical control mice $(p<0.01)$. It is well established by us and others that cognitively normal mice spend $\sim 70 \%$ of their time with the new object compared with the old object (Asuni et al., 2007). The immunized htau/PS1 mice did not differ significantly from their nonimmunized identical control mice in any of the sensorimotor tasks, and all the groups appeared to have normal motor functions based on our experience with wild-type mice in these tests (rotarod, traverse beam, locomotor activity) (see supplemental Fig. 1, available at www.jneurosci. org as supplemental material). These findings indicate that the cognitive improvements observed following the immunization cannot be explained by sensorimotor effects, which further strengthens our results.

Immunohistochemical staining with PHF1 and AT8 antibodies revealed pronounced tau pathology, primarily in the htau/PS1 controls and to a lesser and comparable degree in the other three groups (Fig. 2A-H). PHF1 immunoreactivity was generally more pronounced than AT8 staining, and its quantitative analysis in-

\section{A Antibody Response}
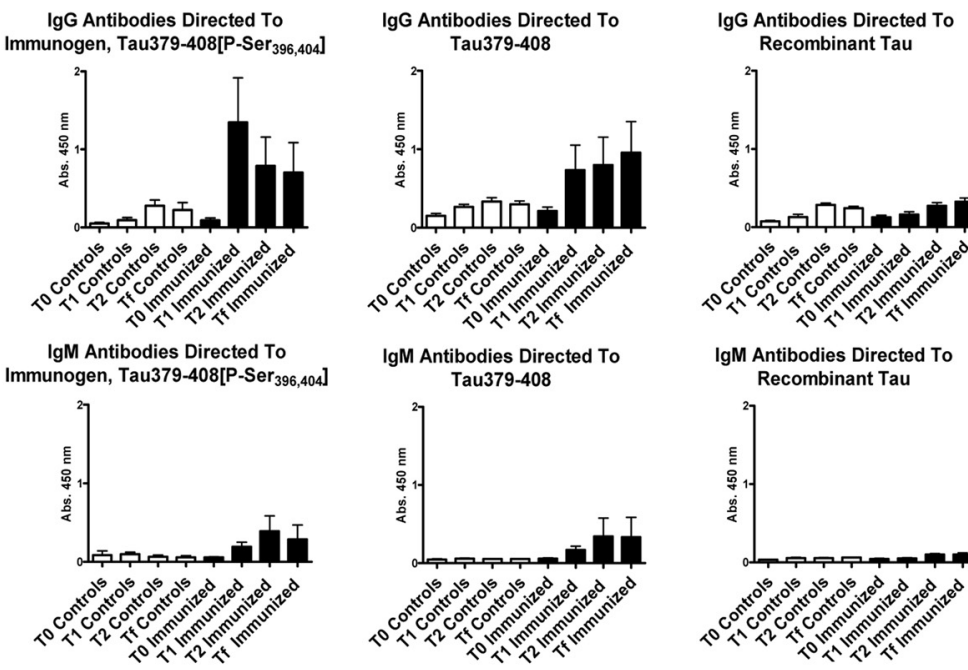

B Radial Arm Maze
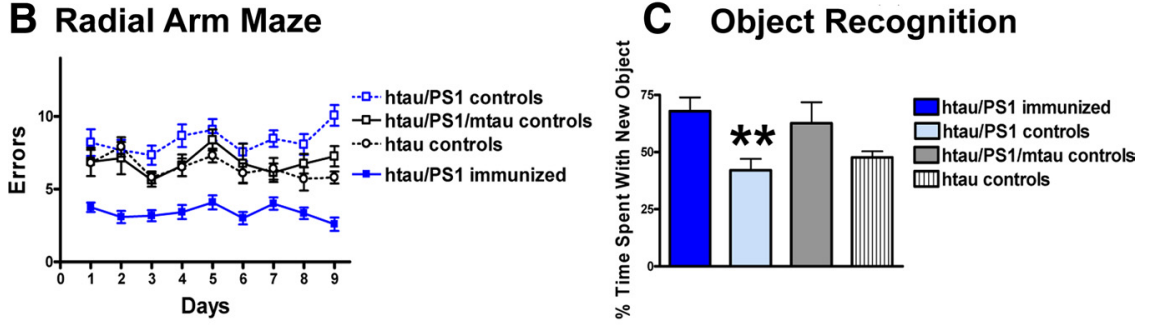

D Closed Field Symmetrical Maze

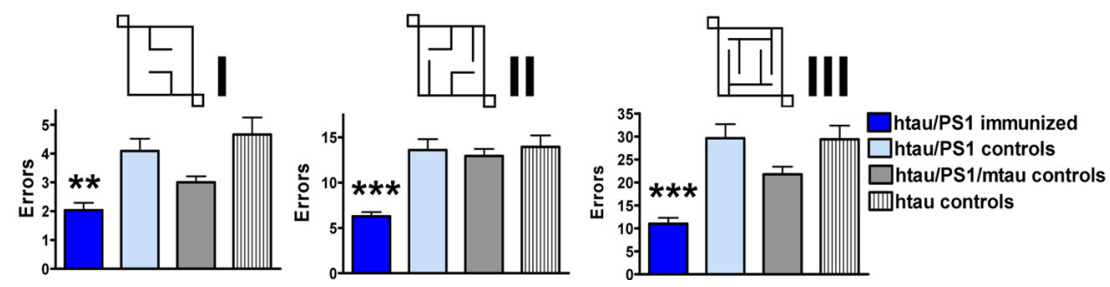

Figure 1. A, The immunogen Tau379 - 408[P-Ser $\left.{ }_{396,404}\right]$ elicits a good lgG antibody response and a modest IgM response. Some autoantibodies are detected in controls and are likely also present in the immunized mice. As expected, because of the overall high immunogenicity of the immunogen, IgG antibodies bind well to the phosphorylated immunogen, Tau379-408[P-Ser ${ }_{396,404}$, as well as its unphosphorylated version, Tau379-408, although a better response is initially generated against the phosphorylated immunogen. Recombinant tau is recognized as well in both controls and immunized mice but to a much lesser degree than the immunogen epitope (phosphorylated or nonphosphorylated). IgM binding is substantially less but has the same pattern as the lgG binding. $\boldsymbol{B}-\boldsymbol{D}$, Immunization with the phosphorylated tau derivative improves performance in three different cognitive tests. $\boldsymbol{B}$, Radial arm maze. Significant difference was observed between the groups in theradial arm maze (two-way ANOVA repeated measures, $p<0.0001$ ). Newman-Keuls posthoctest revealed that the immunized htau/PS1 mice $(n=12)$ performed better than the control htau/PS1 mice $(n=15)$ on all the days $(p<$ 0.01-0.001). Other control groups were htau/PS1/mtau $(n=8)$ and htau $(n=10)$. See Table 1 for gender split. C, Object recognition test. Significant difference was observed between the groups in the object recognition test (one-way ANOVA, $p=0.005, n=8-16$ per group). Newman-Keuls post hoc test revealed that the immunized htau/PS1 mice had better short-term memories than identical control mice $(p<0.01)$. It is well established by us and others that cognitively normal mice spend $\sim 70 \%$ of their time with the new object compared with the old object. ${ }^{* *} p<0.01$. D, Closed field symmetrical maze. Mazes I-III: significant difference was observed between the groups (one-way ANOVA; maze I, $p<0.001$; maze II, $p<0.0001$; maze III, $p<0.01 ; n=7-16$ per group). Post hoc analysis revealed that the treated htau/PS1 group performed better than their identical control mice (mazel, $p<0.01$; mazes II and III, $p<0.001$ ). Posthoc analysis also revealed significant differences between some of the other groups, depending on the maze, but those differences are less relevant and are therefore not detailed here. The three mazes were of increasing complexity, as indicated by the number of errors (note that the $y$-axis scale differs). ${ }^{* *} p<0.01,{ }^{* * *} p<0.001$. Bars or lines represent mean group values plus SEM.

dicated significant differences between the groups (Fig. 2 I) (oneway ANOVA, $p<0.01)$. The therapy reduced PHF1-reactive tau aggregates by $57 \%$ in the pyriform cortex $(p<0.01)$, compared with identical controls. This brain region has previously been chosen for analysis in the htau model (Andorfer et al., 2005) because of its prominent pathology. However, the immunotherapy appeared to reduce tau pathology throughout the brain. The 
Table 1. Number of mice analyzed per group split by gender

\begin{tabular}{|c|c|c|c|c|c|c|c|c|c|c|}
\hline & \multicolumn{2}{|c|}{$\begin{array}{l}\text { Closed field } \\
\text { symmetrical } \\
\text { maze }\end{array}$} & \multicolumn{2}{|c|}{$\begin{array}{l}\text { Object } \\
\text { recognition }\end{array}$} & \multicolumn{2}{|c|}{$\begin{array}{l}\text { Radial arm } \\
\text { maze }\end{array}$} & \multicolumn{2}{|c|}{ Histology } & \multicolumn{2}{|c|}{$\begin{array}{l}\text { Western } \\
\text { blot }\end{array}$} \\
\hline & M & $\mathrm{F}$ & M & $\mathrm{F}$ & M & $\mathrm{F}$ & M & $F$ & M & $\mathrm{F}$ \\
\hline htau/PS1 immunized & 5 & 7 & 5 & 7 & 5 & 7 & 5 & 7 & 4 & 6 \\
\hline htau/PS1 controls & 7 & 9 & 7 & 9 & 7 & 8 & 6 & 4 & 5 & 7 \\
\hline htau/PS1/mtau & 5 & 2 & 5 & 2 & 5 & 3 & 5 & 2 & 5 & 2 \\
\hline htau & 4 & 6 & 4 & 6 & 4 & 6 & 4 & 5 & 4 & 6 \\
\hline
\end{tabular}

M, Males; F, females.

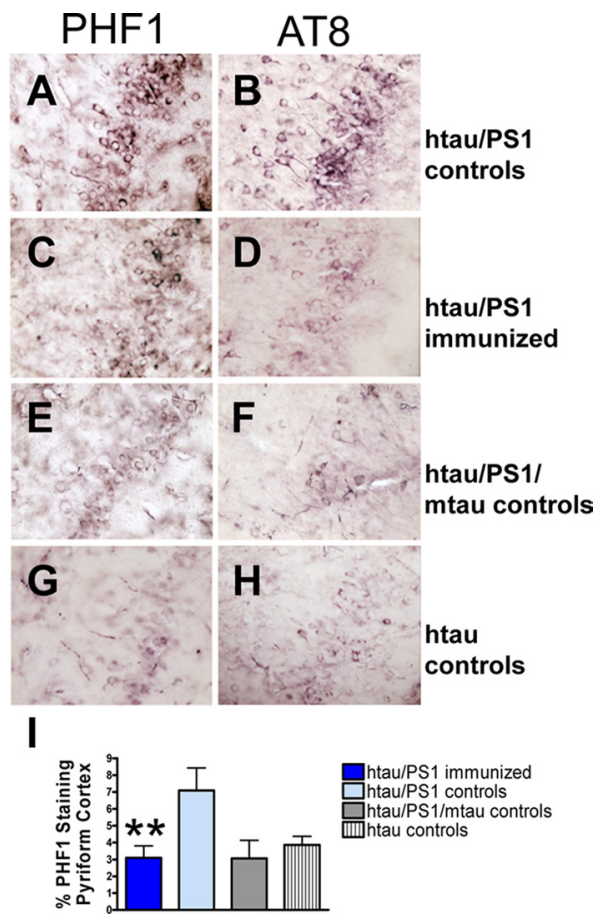

Figure 2. Tau immunotherapy reduces aggregated tau in the brain. $\mathbf{A}-\mathbf{H}$, PHF1 and AT8 immunostaining differed substantially between the groups as depicted here in coronal sections through the pyriform cortex. The degree of PHF1 versus AT8 staining was comparable within groups, although the PHF1 staining was usually more pronounced. $I$, Quantitative analysis of the PHF1 staining revealed a significant difference between the groups (one-way ANOVA, $p<$ $0.01, n=7-12$ per group). Immunized htau/PS1 mice had $57 \%$ less PHF1 immunoreactive tau than their htau/PS1 controls $\left({ }^{* *} p<0.01\right)$. The htau mice had a similar degree of tau pathology as the htau/PS1/mtau mice and the immunized htau/PS1 mice. Bars represent mean group values plus SEM.

htau mice had similar degrees and distributions of tau pathology as the htau/PS1/mtau mice and the immunized htau/PS1 mice. No significant difference was observed between immunized and identical controls in degree of microgliosis [htau/PS1 immunized: $1.7 \pm 0.2$ (average rating \pm SEM); htau/PS1 controls: $1.8 \pm 0.1$ ] or astrogliosis (htau/PS1 immunized: $1.6 \pm 0.1$; htau/ PS1 controls: $1.6 \pm 0.1$ ), suggesting that gradual removal of tau aggregates is not associated with gliosis. In the other control groups, a similar degree of microglial (htau/PS1/mtau: $1.9 \pm 0.1$; htau: $1.4 \pm$ 0.1 ) and astroglial (htau/PS1/mtau: $1.8 \pm 0.1$; htau: $1.8 \pm 0.1$ ) activation was observed. The regional pattern of tau pathology was similar as described previously for the htau model (Andorfer et al., 2003), with prominent cortical and hippocampal involvement, but was more severe in the htau/PS1 model at the age analyzed. A time-course study of the progression of brain pathology in the htau/PS1 model is underway.

For Western blot analysis, total tau was measured with polyclonal B19 antibody, whereas pathological tau was detected with monoclonal PHF1 and CP13 antibodies. Levels of pathological
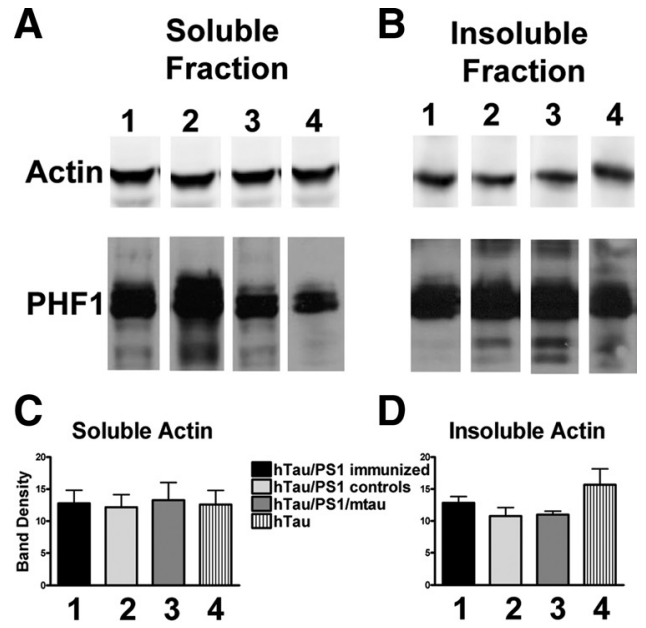

E Soluble PHF1 Tau

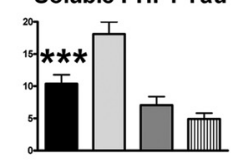

$\mathbf{F}$

Insoluble PHF1 Tau

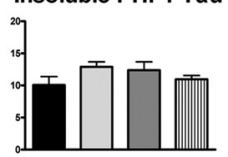

G

Soluble Tau Ratio

H (PHF1/Actin)

Insoluble Tau Ratio

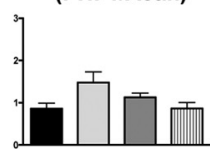

Figure 3. Tau immunotherapy reduces soluble and insoluble PHF1 pathological tau in the brain. $A-D$, Levels of actin in the soluble and insoluble fraction were not altered by immunotherapy and did not differ among the four groups ( $n=7-12$ per group). Gel lanes $1-4$ refer to the same groups that are described in $C$. $\boldsymbol{A}, \boldsymbol{E}$, Levels of soluble PHF1-reactive pathological tau differed among the groups (one-way ANOVA, $p<0.0001$ ) with the control htau/PS1 group differing from the other three groups (Newman-Keuls post hoc test, $p<0.001$ ). Notably, soluble PHF1 levels were reduced by $43 \%$ in the immunized htau/PS1 mice compared with identical controls. G, Overall difference was also observed in the soluble PHF1/actin ratio ( $p=$ 0.01 ), but group differences were less pronounced (Dunn's, htau vs htau/PS1 controls, $p<$ 0.01). There was a strong trend for a reduced PHF1/actin ratio in the immunized htau/PS1 mice compared with identical controls ( $35 \%$ reduction). $\boldsymbol{B}, \boldsymbol{F}, \boldsymbol{H}$, The groups did not differ significantly in their levels of insoluble PHF1 or in insoluble PHF1/actin ratio $(p<0.06)$, but there was a strong trend for reduced levels in immunized htau/PS1 mice compared with their control htau/ PS1 group (PHF1: $22 \%$ reduction; PHF1/actin ratio: $42 \%$ reduction). ${ }^{* * *} p<0.001$. Bars represent mean group values plus SEM.

tau were normalized with actin (Figs. 3, 4) and total tau levels (supplemental Figs. 2, 3, available at www.jneurosci.org as supplemental material). Actin levels did not differ significantly between the groups (Figs. 3, 4A-D), whereas levels of soluble PHF1-stained tau were significantly decreased $(43 \%, p<0.001)$ in the immunized mice compared with their identical controls (Fig. 3E). A trend was observed for a decrease (22\%) in insoluble PHF1-reactive tau (Fig. 3F). Further analysis indicated a very strong trend for the immunotherapy to reduce the ratio of PHF1/ actin by 35 and $42 \%$, respectively, in the soluble and insoluble fractions (Fig. $3 G, H$ ). Likewise, the ratio of $\mathrm{CP} 13 /$ actin in the insoluble fraction was reduced by $29 \%$ (Fig. $4 H$ ). Levels of total soluble and insoluble tau did not differ significantly between the groups (supplemental Fig. $2 A-D$, available at www.jneurosci.org as supplemental material). Further analysis indicated a significant $45 \%$ reduction $(p<0.05)$ in the soluble fraction ratio of PHF1/B19 in the treated group, compared with identical controls, and a very strong trend in the same direction (43\%) in the 


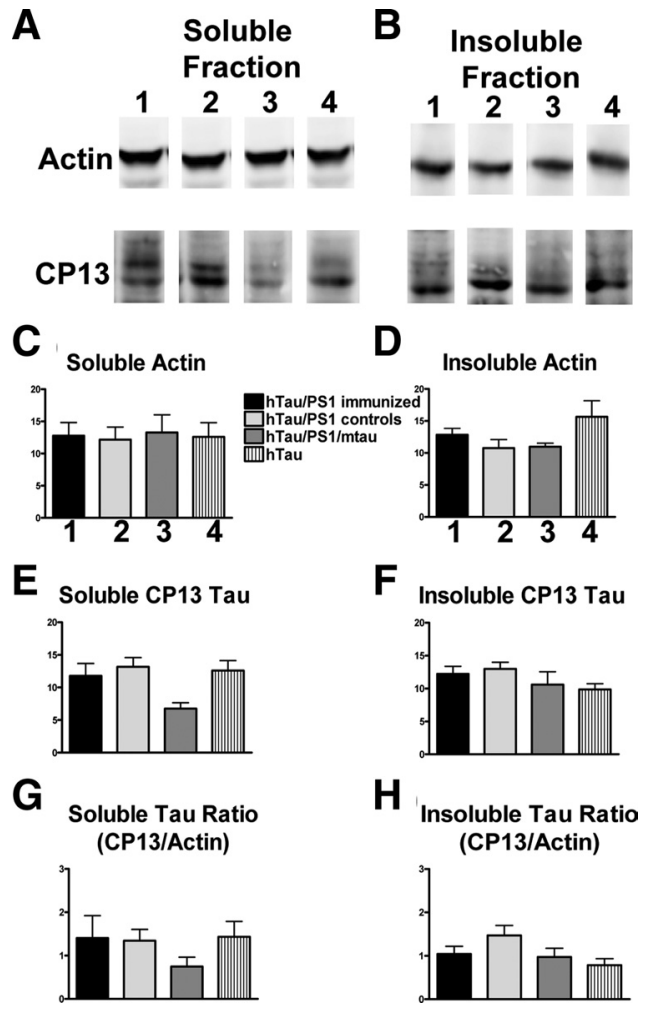

Figure 4. Tau immunotherapy reduces soluble and insoluble CP13 pathological tau in the brain. $\boldsymbol{A}-\boldsymbol{D}$, Levels of actin in the soluble and insoluble fraction were not altered by immunotherapy and did not differ between the four groups ( $n=7-12$ per group). Gel lanes $1-4$ refer to the same groups that are described in $C$. $A, E$, Levels of soluble (P13-reactive pathological tau differed between the groups (one-way ANOVA, $p=0.05$ ) with the control htau/PS1 group differing from the control htau/PS1/mtau group (Newman-Keuls post hoc test, $p<0.05$ ). Notably, soluble CP13 levels were not altered in the immunized htau/PS1 mice compared with identical controls. $G$, Substantially more variance was within each group in the soluble (P13/actin ratio, although the pattern was similar as in $\boldsymbol{E} . \boldsymbol{B}, \boldsymbol{F}, \boldsymbol{H}$, The groups did not differ significantly in their levels of insoluble CP13 or in insoluble CP13/actin ratio $(p<0.08)$, but there was a strong trend for reduced levels in immunized htau/PS1 mice compared with their control htau/PS1 group (CP13/actin ratio: 29\% reduction). Bars represent mean group values plus SEM.

insoluble fraction (supplemental Fig. 2G,H, available at www. jneurosci.org as supplemental material). Likewise, the ratio of CP13/B19 in the insoluble fraction was reduced by 26\% (supplemental Fig. $3 H$, available at www.jneurosci.org as supplemental material). Together, these findings indicate that pathological tau was preferentially being cleared by the immunotherapy.

Importantly, the cognitive improvements correlated well with reduction in PHF1-stained tau aggregates assessed by immunohistochemistry. Significant correlation was observed in all three memory tests [RAM (last day of testing analyzed): $r=0.36, p=$ 0.01; CFSM: maze I, $r=0.33, p=0.02$; maze III, $r=0.40, p=$ 0.01; ORT: $r=-0.31, p=0.03]$. Less consistent correlations were observed between the Western blot fractions and cognitive outcomes that varied depending on the fraction (soluble, insoluble) of tau antibody (PHF1, CP13), the protein used for normalizing the data (total tau, actin), and the cognitive test (data not shown). These findings indicate that tau pathology on histological sections rather than Western blots may predict cognitive outcome. Overall, these results strongly demonstrate the feasibility of tau immunotherapy for $\mathrm{AD}$ and related tauopathies.

\section{Discussion}

We have previously shown the following: (1) antibodies against this immunogen recognize both pathological tau in human AD brain and tangle Tg mouse brain (Asuni et al., 2007); (2) when injected into the carotid artery, these antibodies enter the brain and bind to pathological tau within neurons; and (3) this type of therapy reduces the amount of these aggregates and improves motor performance in a tangle mouse model, JNPL3, containing the P301L tau mutation (Asuni et al., 2007). The severe motor impairments that develop as tau pathology advances in the homozygous JNPL3 mice makes it impossible to thoroughly assess their cognition as this requires extensive maze navigation. Hence, the important question whether tau immunotherapy could prevent cognitive decline remained unanswered. We now show in a new model, htau/PS1, that this type of treatment can indeed prevent cognitive impairments. The htau model does develop spatial memory deficits by 12 months of age, which supports our results and interpretations (Polydoro et al., 2009). Recent findings by others also strengthen the feasibility of tau immunotherapy (Boimel et al., 2010).

Our present findings clearly show a good relationship among antibody titer, the amount of tau aggregates in the brain, and performance on various cognitive tasks, indicating that anti-tau antibodies have at least a major role in the therapeutic outcome. As tau is mostly found intracellularly, it has been an elusive target. However, numerous studies have shown neuronal uptake of antibodies, and it appears to be accelerated under pathological conditions (Sigurdsson, 2008). Furthermore, other intracellular protein aggregates, $\alpha$-synuclein and A $\beta$, can be targeted by immunotherapy (Masliah et al., 2005b; Tampellini et al., 2007). Clearance of extracellular tau may also reduce associated pathology, and indirectly facilitate removal of intracellular tau (Sigurdsson, 2009), as it may be important for the spread of tau pathology throughout the brain (Clavaguera et al., 2009; Frost et al., 2009). Previously, tau has been reported to be secreted in cell culture and to have an extracellular function (Gómez-Ramos et al., 2008), which may become aberrant if it accumulates. Together, these reports and our prior and current findings provide strong support for the feasibility of harnessing the immune system to target and clear pathological tau in $\mathrm{AD}$ and related tauopathies.

Normal tau and at least certain species of nonaggregated hyperphosphorylated tau are likely degraded by the proteosome system as other soluble proteins (Petrucelli et al., 2004), whereas upon aggregation under pathological conditions, clearance through the autophagy/lysosomal system should be favored. Indeed, prevalent lysosomal and autophagic vesicles have been detected by ultrastructural analysis in the JNPL3 P301L tangle mouse model (Lin et al., 2003), as well as in neuronal cultures that express various tau mutations (Lim et al., 2001). Interestingly, early pathological changes have been observed in the lysosomal system in AD (Nixon, 2007), which may be a primary event of unknown origin or secondary to intracellular aggregation of $\mathrm{A} \beta$ and/or tau. Regardless of the exact cause, antibody-mediated disassembly of these aggregates should facilitate their lysosomal clearance and thereby promote neuronal health. In further support of this scenario, lysosomal tau is detected in $\mathrm{AD}$ and control brains (Ikeda et al., 1998), inhibition of lysosomes increases tau levels (Bendiske and Bahr, 2003), macroautophagy is likely involved in tau clearance (Berger et al., 2006; Hamano et al., 2008), and lysosomal processing has most recently been shown to influence tau aggregation and clearance in an inducible cellular model of tauopathy (Wang et al., 2009). Furthermore, antibodies have been visualized in lysosomes by immunoelectroscopy (Meeker et al., 1987).

Potential side effects of tau immunotherapy could be associated with clearance of normal tau but total tau levels were not reduced in the treated animals. Astrogliosis, a sensitive indicator of neurotoxicity, did not appear to be increased in the immunized 
mice, further supporting the safety of the approach. As the antibodies will mainly clear extracellular tau aggregates and/or be primarily taken up into diseased neurons with accumulated tau, those entities should be preferentially targeted. This clearance was not associated with enhanced microgliosis, which may be explained by gradual removal over an extended period. Intracellularly, the antibodies would be processed through the endosomal/autophagic/lysosomal system and, therefore, solely interact with tau aggregates clogging the same pathway, whereas normal soluble tau would primarily be located in the cytosol and hence not accessible. However, some clearance of healthy tau may not be detrimental as tau knock-out animals appear remarkably normal, indicating that other microtubule associated protein(s) can perform similar functions (Denk and Wade-Martins, 2009). Furthermore, reducing normal endogenous tau has been shown to ameliorate $\mathrm{A} \beta$-induced dysfunction in transgenic mice (Roberson et al., 2007).

It is not known how the clearance of tau pathology influences $\mathrm{A} \beta$, but immunotherapeutic clearance of $\mathrm{A} \beta$ in humans or mice has modest effect on tau pathology (Oddo et al., 2004; Boche et al., 2010a,b; Serrano-Pozo et al., 2010), and CSF levels of tau were reported to be reduced in antibody responders in the halted AN1792 trial (Gilman et al., 2005). For cognitive improvement in A $\beta$ mouse models, it seems more important to remove early-stage $\mathrm{A} \beta$ assemblies than plaques (Lambert et al., 1998). A similar concept may apply to tau pathology, as suppression of transgenic tau expression improves memory in a mouse model despite stable levels of neurofibrillary tangles (NFTs) (Santacruz et al., 2005), and synaptic loss precedes tangle formation in a different mouse model (Yoshiyama et al., 2007). Together, these findings support that clearing early-stage pathological tau is likely beneficial, and these smaller intracellular and extracellular assemblies should also be easier to clear than late-stage NFTs. We are currently assessing whether tau pathology can be reversed in older mice.

The PS1 M146L model we used to generate the htau/PS1 mice does not develop tangles, at least up to 8 months of age, when bred with Tg2576 A $\beta$ plaque mice (Holcomb et al., 1998), but tau hyperphosphorylation has been reported in this APP/PS1 cross at 6 months (Kurt et al., 2003). Other studies have shown tau hyperphosphorylation in PS1 I213T knock-in mice that becomes evident in the hippocampus at $\sim 7$ months (Tanemura et al., 2006), and in the spinal cord of PS1 $1 \mathrm{E} 9$ mice at 5 months (Lazarov et al., 2007). Additional models that express one isoform of wild-type human tau (0N3R) and PS1 M146L with or without APP mutations (Boutajangout et al., 2002, 2004), show a somatodendritic accumulation of phosphorylated tau without tangle formation that is not enhanced by $\mathrm{A} \beta$ plaque development. Overall, the tau pathology in these models appears to be less than in the htau/PS1 cross that we generated on a mouse tau knockout background. These differences can be explained by their expression of mouse tau that seems to interfere with aggregation of human tau (Andorfer et al., 2003), as well as limited if any expression of human tau isoforms. Autopsy findings are more variable with some studies, suggesting that presenilin mutations may enhance tangle pathology compared with sporadic $\mathrm{AD}$ whereas others show no difference (Shepherd et al., 2009). The exact mechanism for how PS1 mutations may enhance tau phosphorylation is not known, but it is likely to involve GSK-3 $\beta$. PS1 can form a complex with this enzyme (Takashima et al., 1998; Tesco and Tanzi, 2000), and PS1 mutations have been shown to increase GSK-3 $\beta$ activity in cultures and animal models (Takashima et al., 1998; Weihl et al., 1999; Pigino et al., 2003; Baki et al., 2004; Tanemura et al., 2006).
Overall, we have demonstrated that immunotherapy targeting pathological tau reduces its aggregates within the brain in a novel AD tangle mouse model, and importantly prevents cognitive deterioration, indicating that it has a great potential as a therapy for $\mathrm{AD}$ and related tauopathies.

\section{References}

Andorfer C, Kress Y, Espinoza M, de Silva R, Tucker KL, Barde YA, Duff K, Davies P (2003) Hyperphosphorylation and aggregation of tau in mice expressing normal human tau isoforms. J Neurochem 86:582-590.

Andorfer C, Acker CM, Kress Y, Hof PR, Duff K, Davies P (2005) Cell-cycle reentry and cell death in transgenic mice expressing nonmutant human tau isoforms. J Neurosci 25:5446-5454.

Asuni AA, Boutajangout A, Scholtzova H, Knudsen E, Li YS, Quartermain D, Frangione B, Wisniewski T, Sigurdsson EM (2006) Vaccination of Alzheimer's model mice with $\mathrm{A} \beta$ derivative in alum adjuvant reduces $\mathrm{A} \beta$ burden without microhemorrhages. Eur J Neurosci 24:2530-2542.

Asuni AA, Boutajangout A, Quartermain D, Sigurdsson EM (2007) Immunotherapy targeting pathological tau conformers in a tangle mouse model reduces brain pathology with associated functional improvements. J Neurosci 27:9115-9129.

Baki L, Shioi J, Wen P, Shao Z, Schwarzman A, Gama-Sosa M, Neve R, Robakis NK (2004) PS1 activates PI3K thus inhibiting GSK-3 activity and tau overphosphorylation: effects of FAD mutations. EMBO J 23:2586-2596.

Bendiske J, Bahr BA (2003) Lysosomal activation is a compensatory response against protein accumulation and associated synaptopathogenesis_-an approach for slowing Alzheimer disease? J Neuropathol Exp Neurol 62:451-463.

Berger Z, Ravikumar B, Menzies FM, Oroz LG, Underwood BR, Pangalos MN, Schmitt I, Wullner U, Evert BO, O’Kane CJ, Rubinsztein DC (2006) Rapamycin alleviates toxicity of different aggregate-prone proteins. Hum Mol Genet 15:433-442.

Boche D, Denham N, Holmes C, Nicoll JA (2010a) Neuropathology after active A $\beta 42$ immunotherapy: implications for Alzheimer's disease pathogenesis. Acta Neuropathol 120:369-384.

Boche D, Donald J, Love S, Harris S, Neal JW, Holmes C, Nicoll JA (2010b) Reduction of aggregated Tau in neuronal processes but not in the cell bodies after A $\beta 42$ immunisation in Alzheimer's disease. Acta Neuropathol 120:13-20.

Boimel M, Grigoriadis N, Lourbopoulos A, Haber E, Abramsky O, Rosenmann $H$ (2010) Efficacy and safety of immunization with phosphorylated tau against neurofibrillary tangles in mice. Exp Neurol 224:472-485.

Boutajangout A, Leroy K, Touchet N, Authelet M, Blanchard V, Tremp G, Pradier L, Brion JP (2002) Increased tau phosphorylation but absence of formation of neurofibrillary tangles in mice double transgenic for human tau and Alzheimer mutant (M146L) presenilin-1. Neurosci Lett 318:29-33.

Boutajangout A, Authelet M, Blanchard V, Touchet N, Tremp G, Pradier L, Brion JP (2004) Characterisation of cytoskeletal abnormalities in mice transgenic for wild-type human tau and familial Alzheimer's disease mutants of APP and presenilin-1. Neurobiol Dis 15:47-60.

Clavaguera F, Bolmont T, Crowther RA, Abramowski D, Frank S, Probst A, Fraser G, Stalder AK, Beibel M, Staufenbiel M, Jucker M, Goedert M, Tolnay M (2009) Transmission and spreading of tauopathy in transgenic mouse brain. Nat Cell Biol 11:909-913.

Denk F, Wade-Martins R (2009) Knock-out and transgenic mouse models of tauopathies. Neurobiol Aging 30:1-13.

Duff K, Eckman C, Zehr C, Yu X, Prada CM, Perez-tur J, Hutton M, Buee L, Harigaya Y, Yager D, Morgan D, Gordon MN, Holcomb L, Refolo L, Zenk B, Hardy J, Younkin S (1996) Increased amyloid- $\beta$ 42(43) in brains of mice expressing mutant presenilin 1. Nature 383:710-713.

Ferrer I, Boada Rovira M, Sánchez Guerra ML, Rey MJ, Costa-Jussá F (2004) Neuropathology and pathogenesis of encephalitis following amyloid- $\beta$ immunization in Alzheimer's disease. Brain Pathol 14:11-20.

Frost B, Jacks RL, Diamond MI (2009) Propagation of tau misfolding from the outside to the inside of a cell. J Biol Chem 284:12845-12852.

Gilman S, Koller M, Black RS, Jenkins L, Griffith SG, Fox NC, Eisner L, Kirby L, Rovira MB, Forette F, Orgogozo JM (2005) Clinical effects of A $\beta$ immunization (AN1792) in patients with AD in an interrupted trial. Neurology 64:1553-1562.

Goedert M, Jakes R, Crowther RA, Six J, Lübke U, Vandermeeren M, Cras P, 
Trojanowski JQ, Lee VM (1993) The abnormal phosphorylation of tau protein at Ser-202 in Alzheimer disease recapitulates phosphorylation during development. Proc Natl Acad Sci U S A 90:5066-5070.

Gómez-Ramos A, Díaz-Hernández M, Rubio A, Miras-Portugal MT, Avila J (2008) Extracellular tau promotes intracellular calcium increase through M1 and M3 muscarinic receptors in neuronal cells. Mol Cell Neurosci $37: 673-681$.

Hamano T, Gendron TF, Causevic E, Yen SH, Lin WL, Isidoro C, Deture M, Ko LW (2008) Autophagic-lysosomal perturbation enhances tau aggregation in transfectants with induced wild-type tau expression. Eur J Neurosci 27:1119-1130.

Holcomb L, Gordon MN, McGowan E, Yu X, Benkovic S, Jantzen P, Wright K, Saad I, Mueller R, Morgan D, Sanders S, Zehr C, O'Campo K, Hardy J, Prada CM, Eckman C, Younkin S, Hsiao K, Duff K (1998) Accelerated Alzheimer-type phenotype in transgenic mice carrying both mutant amyloid precursor protein and presenilin 1 transgenes. Nat Med 4:97-100.

Holmes C, Boche D, Wilkinson D, Yadegarfar G, Hopkins V, Bayer A, Jones RW, Bullock R, Love S, Neal JW, Zotova E, Nicoll JA (2008) Long-term effects of A $\beta 42$ immunisation in Alzheimer's disease: follow-up of a randomised, placebo-controlled phase I trial. Lancet 372:216-223.

Ikeda K, Akiyama H, Arai T, Kondo H, Haga C, Iritani S, Tsuchiya K (1998) Alz-50/Gallyas-positive lysosome-like intraneuronal granules in Alzheimer's disease and control brains. Neurosci Lett 258:113-116.

Kerchner GA, Boxer AL (2010) Bapineuzumab. Expert Opin Biol Ther 10:1121-1130.

Kurt MA, Davies DC, Kidd M, Duff K, Howlett DR (2003) Hyperphosphorylated tau and paired helical filament-like structures in the brains of mice carrying mutant amyloid precursor protein and mutant presenilin-1 transgenes. Neurobiol Dis 14:89-97.

Lambert MP, Barlow AK, Chromy BA, Edwards C, Freed R, Liosatos M, Morgan TE, Rozovsky I, Trommer B, Viola KL, Wals P, Zhang C, Finch CE, Krafft GA, Klein WL (1998) Diffusible, nonfibrillar ligands derived from A $\beta 1-42$ are potent central nervous system neurotoxins. Proc Natl Acad Sci U S A 95:6448-6453.

Lazarov O, Morfini GA, Pigino G, Gadadhar A, Chen X, Robinson J, Ho H, Brady ST, Sisodia SS (2007) Impairments in fast axonal transport and motor neuron deficits in transgenic mice expressing familial Alzheimer's disease-linked mutant presenilin 1. J Neurosci 27:7011-7020.

Lim F, Hernández F, Lucas JJ, Gómez-Ramos P, Morán MA, Avila J (2001) FTDP-17 mutations in tau transgenic mice provoke lysosomal abnormalities and tau filaments in forebrain. Mol Cell Neurosci 18:702-714.

Lin WL, Lewis J, Yen SH, Hutton M, Dickson DW (2003) Ultrastructural neuronal pathology in transgenic mice expressing mutant (P301L) human tau. J Neurocytol 32:1091-1105.

Masliah E, Hansen L, Adame A, Crews L, Bard F, Lee C, Seubert P, Games D, Kirby L, Schenk D (2005a) A $\beta$ vaccination effects on plaque pathology in the absence of encephalitis in Alzheimer disease. Neurology 64:129-131.

Masliah E, Rockenstein E, Adame A, Alford M, Crews L, Hashimoto M, Seubert P, Lee M, Goldstein J, Chilcote T, Games D, Schenk D (2005b) Effects of $\alpha$-synuclein immunization in a mouse model of Parkinson's disease. Neuron 46:857-868.

Meeker ML, Meeker RB, Hayward JN (1987) Accumulation of circulating endogenous and exogenous immunoglobulins by hypothalamic magnocellular neurons. Brain Res 423:45-55.

Nicoll JA, Wilkinson D, Holmes C, Steart P, Markham H, Weller RO (2003) Neuropathology of human Alzheimer disease after immunization with amyloid- $\beta$ peptide: a case report. Nat Med 9:448 -452 .

Nixon RA (2007) Autophagy, amyloidogenesis and Alzheimer disease. J Cell Sci 120:4081-4091.

Oddo S, Billings L, Kesslak JP, Cribbs DH, LaFerla FM (2004) A $\beta$ immunotherapy leads to clearance of early, but not late, hyperphosphorylated tau aggregates via the proteasome. Neuron 43:321-332.

Otvos L Jr, Feiner L, Lang E, Szendrei GI, Goedert M, Lee VM (1994) Monoclonal antibody PHF-1 recognizes tau protein phosphorylated at serine residues 396 and 404. J Neurosci Res 39:669-673.

Petrucelli L, Dickson D, Kehoe K, Taylor J, Snyder H, Grover A, De Lucia M, McGowan E, Lewis J, Prihar G, Kim J, Dillmann WH, Browne SE, Hall A, Voellmy R, Tsuboi Y, Dawson TM, Wolozin B, Hardy J, Hutton M (2004) CHIP and Hsp70 regulate tau ubiquitination, degradation and aggregation. Hum Mol Genet 13:703-714.
Pigino G, Morfini G, Pelsman A, Mattson MP, Brady ST, Busciglio J (2003) Alzheimer's presenilin 1 mutations impair kinesin-based axonal transport. J Neurosci 23:4499-4508.

Polydoro M, Acker CM, Duff K, Castillo PE, Davies P (2009) Agedependent impairment of cognitive and synaptic function in the htau mouse model of tau pathology. J Neurosci 29:10741-10749.

Roberson ED, Scearce-Levie K, Palop JJ, Yan F, Cheng IH, Wu T, Gerstein H, Yu GQ, Mucke L (2007) Reducing endogenous tau ameliorates amyloid $\beta$-induced deficits in an Alzheimer's disease mouse model. Science 316:750-754.

Sadowski M, Pankiewicz J, Scholtzova H, Ji Y, Quartermain D, Jensen CH, Duff K, Nixon RA, Gruen RJ, Wisniewski T (2004) Amyloid- $\beta$ deposition is associated with decreased hippocampal glucose metabolism and spatial memory impairment in APP/PS1 mice. J Neuropathol Exp Neurol 63:418-428.

Santacruz K, Lewis J, Spires T, Paulson J, Kotilinek L, Ingelsson M, Guimaraes A, DeTure M, Ramsden M, McGowan E, Forster C, Yue M, Orne J, Janus C, Mariash A, Kuskowski M, Hyman B, Hutton M, Ashe KH (2005) Tau suppression in a neurodegenerative mouse model improves memory function. Science 309:476-481.

Serrano-Pozo A, William CM, Ferrer I, Uro-Coste E, Delisle MB, Maurage CA, Hock C, Nitsch RM, Masliah E, Growdon JH, Frosch MP, Hyman BT (2010) Beneficial effect of human anti-amyloid- $\beta$ active immunization on neurite morphology and tau pathology. Brain 133:1312-1327.

Shepherd C, McCann H, Halliday GM (2009) Variations in the neuropathology of familial Alzheimer's disease. Acta Neuropathol 118:37-52.

Sigurdsson EM (2008) Immunotherapy targeting pathological tau protein in Alzheimer's disease and related tauopathies. J Alzheimers Dis 15:157-168.

Sigurdsson EM (2009) Tau-focused immunotherapy for Alzheimer's disease and related tauopathies. Curr Alzheimer Res 6:446-450.

Sigurdsson EM, Lorens SA, Hejna MJ, Dong XW, Lee JM (1996) Local and distant histopathological effects of unilateral amyloid- $\beta$ 25-35 injections into the amygdala of young F344 rats. Neurobiol Aging 17:893-901.

Sigurdsson EM, Scholtzova H, Mehta PD, Frangione B, Wisniewski T (2001) Immunization with a nontoxic/nonfibrillar amyloid- $\beta$ homologous peptide reduces Alzheimer's disease associated pathology in transgenic mice. Am J Pathol 159:439-447.

Sigurdsson EM, Knudsen E, Asuni A, Fitzer-Attas C, Sage D, Quartermain D, Goni F, Frangione B, Wisniewski T (2004) An attenuated immune response is sufficient to enhance cognition in an Alzheimer's disease mouse model immunized with amyloid- $\beta$ derivatives. J Neurosci 24:6277-6282.

Takashima A, Murayama M, Murayama O, Kohno T, Honda T, Yasutake K, Nihonmatsu N, Mercken M, Yamaguchi H, Sugihara S, Wolozin B (1998) Presenilin 1 associates with glycogen synthase kinase- $3 \beta$ and its substrate tau. Proc Natl Acad Sci U S A 95:9637-9641.

Tampellini D, Magrané J, Takahashi RH, Li F, Lin MT, Almeida CG, Gouras GK (2007) Internalized antibodies to the A $\beta$ domain of APP reduce neuronal $\mathrm{A} \beta$ and protect against synaptic alterations. J Biol Chem 282:18895-18906.

Tanemura K, Chui DH, Fukuda T, Murayama M, Park JM, Akagi T, Tatebayashi Y, Miyasaka T, Kimura T, Hashikawa T, Nakano Y, Kudo T, Takeda M, Takashima A (2006) Formation of tau inclusions in knock-in mice with familial Alzheimer disease (FAD) mutation of presenilin 1 (PS1). J Biol Chem 281:5037-5041.

Tesco G, Tanzi RE (2000) GSK3 $\beta$ forms a tetrameric complex with endogenous PS1-CTF/NTF and $\beta$-catenin. Effects of the D257/D385A and FAD-linked mutations. Ann N Y Acad Sci 920:227-232.

Wang Y, Martinez-Vicente M, Krüger U, Kaushik S, Wong E, Mandelkow EM, Cuervo AM, Mandelkow E (2009) Tau fragmentation, aggregation and clearance: the dual role of lysosomal processing. Hum Mol Genet 18:4153-4170.

Weihl CC, Ghadge GD, Kennedy SG, Hay N, Miller RJ, Roos RP (1999) Mutant presenilin-1 induces apoptosis and downregulates Akt/PKB. J Neurosci 19:5360-5369.

Yoshiyama Y, Higuchi M, Zhang B, Huang SM, Iwata N, Saido TC, Maeda J, Suhara T, Trojanowski JQ, Lee VM (2007) Synapse loss and microglial activation precede tangles in a P301S tauopathy mouse model. Neuron 53:337-351. 